\title{
Subsurface metals fatigue cracking without and with crack tip
}

\author{
Andrey Shanyavskiy \\ State Centre for Flights Safety, Airport Sheremetievo-1, PO Box 54, Moscow region, Chimkinskiy State, 141426, Russia, \\ shananta@mailfrom.ru
}

\begin{abstract}
Very-High-Cycle-Fatigue regime for metals was considered and mechanisms of the subsurface crack origination were introduced. In many metals first step of crack origination takes place with specific area formation because of material pressing and rotation that directed to transition in any volume to material ultrahigh-plasticity with nano-structure appearing. Then by the border of the nano-structure takes place volume rotation and fracture surface creates with spherical particles which usually named Fine-Granular-Area. In another case there takes place First-Smooth-Facet occurring in area of origin due to whirls appearing by the one of the slip systems under discussed the same stress-state conditions. Around Fine-Granular-Area or FirstSmooth-Facet there plastic zone appeared and, then, subsurface cracking develops by the same manner as for through cracks. In was discussed quantum-mechanical nature of fatigue crack growth in accordance with Yang's modulus quantization for low level of deformations. New simply equation was considered for describing subsurface cracking in metals out of Fine-Granular-Area or Fist-Smooth-Facet.
\end{abstract}

KEYwORDS. Very-High-Cycle-Fatigue; Subsurface; Mechanisms; Fine-granular-area; First-smooth-facet; Ultrahigh-plasticity; Quantum-mechanical; Crack growth.

\section{INTRODUCTION}

$\mathrm{F}$ atigue crack growth for metals subsurface cracking in VHCF regime has been investigated in many cases [1-5]. Main idea is about continuous (conventional) fatigue crack propagation process in spite of registered range of increments of crack in one cycle in two orders less than crystallographic lattice. It is physically clear that crack increment cannot be less than one lattice. That is why crack growth process in the considered case is not continuous.

Another basically idea is about subsurface cracking process that takes place in vacuum [6]. Special tests in vacuum have been performed for steels and titanium alloys and it was demonstrated similarity in fracture surface patterns for through and subsurface cracks when through cracks did propagation in vacuum. Moreover, it was discovered Fine-Granular-Area (FGA) for the first stage of through crack growth in vacuum.

The idea about subsurface fatigue crack propagation in vacuum contradicted with test results of steels having different contents of hydrogen [7]. If hydrogen contents in metals has increasing, subsurface crack initiation has acceleration. Clear that, if hydrogen influenced metals cracking, there is subsurface environment for developed fatigue cracks. In the center of a fish-eye area there has been discovered high level of oxygen $[8,9]$. This is the same evidence that subsurface cracking can takes place in oxygen environment.

In many cases studied of steels [10] and superalloy [8] subsurface cracking, there were very clear registered fatigue striations on the fracture surface which has been formed before crack evidence on the specimen surface. The fatigue striation formation process has been considered based on the knowledge about environment influence on this process. This consideration based on well-known investigation results which show that fatigue striations have not evidence in vacuum. Consequently, the discussed investigation results demonstrate that subsurface cracking occurs in environment but not in vacuum. 
It should be corrected knowledge about meaning of the word that used to such indication environment state as "vacuum". Usually considered: (1) low vacuum with gas pressure less than $100 \mathrm{~Pa}$; (2) middle pressure with $0.1<\mathrm{p}$ $<100 \mathrm{~Pa}$; (3) high vacuum with 10-5 $\mathrm{Pa}<\mathrm{p}<0.1 \mathrm{~Pa}$; (4) ultra-high-vacuum with $\mathrm{p}<10-5 \mathrm{~Pa}$.

Tests in vacuum performed under continual pumping with vacuum pumps to support needed level of low pressure in specimen chamber but not less that $10^{-5} \mathrm{~Pa}$. At the same time it should be clear that always in specimen chamber exist pressure of gases. This situation attests itself as dynamical vacuum. It means that inside of camera when tests perform exists low pressure always. That is why it should be corrected meaning of word "vacuum': it is environment with low pressure of gases or other elements.

The problem of subsurface crack initiation and propagation has been briefly discussed in the case of titanium alloy VT3-1 [11]. Following by this consideration was clear that subsurface fatigue cracking performs not in vacuum but in low level pressure of rest cases existing in metals which are enough for environment influence consideration on this process.

Moreover, investigating of crack initiation processes, occurring and developing in chemically clean metals because of different types of defects formation, such as, for instance, pore's, microcracks and etc., the theory of strength suggested that there inside of the defect volume has to be vacuum state. But it contradicted with physics of phase transformation [12]. From the diagram of phase transformation for each chemical element followed that solid phase cannot be in equilibrium state at the contact with vacuum. The solid body equilibrium state can be possible, if exist interaction effect between its own vapor or liquid in the triple point, T3, where simultaneously exist three phases. For example, if there exists in solid body spherical pore, it needs to be fill up of vapor or liquid metal. If volume of pores will be increased under external stressing, quantity of liquid and gas phases also will have increase.

Nevertheless, but it is clear that subsurface fatigue cracking in metals takes place not in vacuum but in specific environment having low value of rest gases or other elements pressure.

At the surface layer atomic stress-state is not the same that subsurface. There is biaxial stress-state at the matrix by the surface and hydrostatic case takes place subsurface. That is why energy dissipation at the surface is more effective than subsurface. Moreover, at the surface takes place disordering in atomic state. That is why there is more effective energy dissipation process under low level of deformation than subsurface.

Subsurface hydrostatic state influenced not only residual state because of non-elastic deformation but there metals can have transition in Ultra-High-Plasticity (UHP) state that has been discussed in the first part of our paper. Also, there can be developed deformations not only during subsurface crack origination because of material volume rotations [11] but for crack propagation too. So, it should be considered metals critical state for crack origination and propagation by the three criteria: (1) non-elastic local deformation; (2) whirls formation under hydrostatic stress-state; (3) self-organized introducing in area of cracked metals own vapor or liquid because free surface can be creates only in environment conditions with some pressure.

Below will be discussed process of subsurface fatigue crack propagation in steels in gigacycle or Ultra-High-Cycle-Fatigue (UHCF) regime based on the introduced above criteria.

\section{MECHANISM OF FGA FORMING}

$\mathrm{W}$ e will discuss process of FGA formation during crack propagation based on discovered fracture surface patterns for investigated steels $[2,13]$.

Typical fracture surface patterns for all specimens are shown in Fig.1. There are spherical and elliptically shaped particles and the same shape has roughed fracture surface in FGA. It is not granules. It is nano-structure of metals that has been performed during cyclic loading. In some FGA places there very clear seen "dimples" because several particles have been moved from their position during deformation process during crack propagation.

Size of particles is not more than $50 \mathrm{~nm}$. It is typical nano-structure for investigated metals. Investigations subsurface layers in the depth direction for one and another part of fracture surfaces in FGA discovered there the same ensemble of particles which structure has difference from matrix structure [2].

Chemical composition investigations of the FGA in steels performed by the fracture surface have shown that there is more percent of carbon than exists in metals composition [14]. Following consideration of non-elastic diffusion in metals this effect can be clear explained. During metals plastic deformation in the case of hydrostatic pressure at the crack tip there takes place local matrix heating for propagating crack, carbides destruction, and, in another way, carbon from matrix has possibility to move inside of free area for occurred crack, and carbon moves there during crack propagation. This effect excluded ultra-high-vacuum state inside of the growing crack. Because of hydrostatic stress-state the diffusion process can be considered based on the model that has been introduced for explanation subsurface crack occurring in the 
case of rolling contact [15]. This case also related to hydrostatic pressing. For the introduced consideration in discussed case of external loading condition, the carbon diffusion in crack takes place because of carbides destruction. Now it is clear that the carbon diffusion in area of crack is main process for steels that followed subsurface cracking.
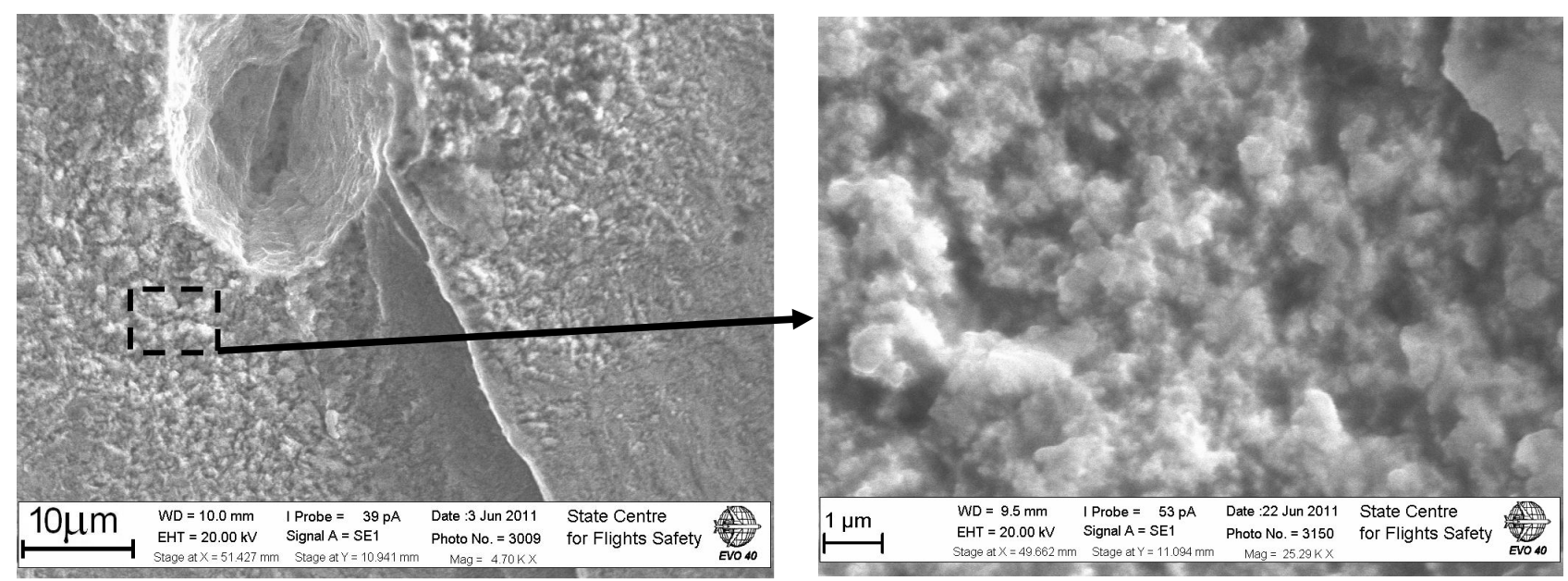

Figure 1: View of Fine-Granular-Area under high magnification [13]. Spherical particles are dominant of the surface pattern.

Let be discussed metals composition in the depth direction in FGA. It is clear that the same spheres or ellipsoids that have been discovered by the fracture surface followed metals subsurface cracking in the depth direction from the crack edges [2]. This type of nano-structures cannot be suddenly appeared in metals. It should be developed in time some process of their formation in the thin layer of FGA where UHP has taken place. Then in matrix develops specific process of fracture surface formation by the border of this structure. This fracture mechanism ought to be considered as the one of the step in nano-structure formation process based on earlier introduced consideration of spherical and elliptically shaped particles formation during fatigue crack propagation $[16,17]$.

Earlier the same type of fracture surfaces patterns as ellipsoidal and spherical particles have been discovered in Al-based alloys subjected to biaxial cyclic loading. The spherical or ellipsoidal particles had occurring because of specific process of metals deformation and destruction under local rotation when intensive pressing of material volume is occurred. In all cases of metal cracking under cyclic loads there has non-conventional manner of matrix fracture along the crack front.

In the discussed case of FGA formation for steels or other metals there is appearance of the same particles formation mechanism, Fig2.

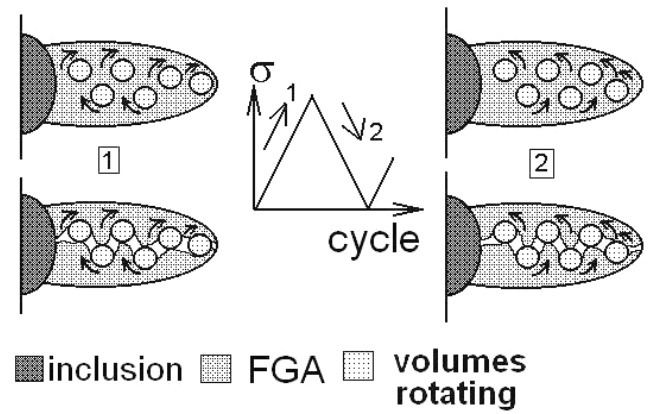

Figure 2: Schema of the spherical particles formation in FGA origin after ultra-high-plasticity in some metal's volume

After metals experienced UHP in area of the crack origination, inside of the plastically deformed volume there starts the rotation deformation process of local volumes inside the area of UHP. If critical density of defects is exceeded, by the border of rotated volumes appeared in homogeneity with spherical shape of fracture surface formation and with spherically or elliptically shaped particles too. During rotations in volume which will be in a further failed with FGA formation, there takes place heating and inside of the volume can go carbon, rest gases and other elements of decomposed matrix. 
The discussed process of particles formation directed to crack growth retardation [16]. That is why cracking of metals during FGA formation cannot be strongly expressed in term of crack growth rates now because it is not conventional process. It can be only say that the estimated crack growth in area out of FGA is less than all period of subsurface metals cracking from the moment of start to form FGA.

Based on the performed consideration, it can be summarized process of FGA formation during metals subsurface fatigue cracking in the next manner: (1) In steels under cyclic loads there in the area of UHP takes place diffusion carbon with thin layers formation by the occurred free surface. The main role in fracture surface formation played matrix rotation instability that directed to for spherical particles and spherical in shape surface patterns. Carbon is good seal for preventing crack edges interaction effect that is why its moves in area of the crack. Hydrogen influenced this process being in matrix and trapped inclusions; (2) In titanium alloys there takes place diffusion of rest gases inside of the crack area and, also, some of the matrix chemical components can go in [11].

Based on the considered sequence of fatigue crack growth events we can conclude that in all discussed cases of steels fatigue cracking there were four areas of different processes of fracture surface formation: (1) Crack origination because of formation FGA, FSF, or Supergrain cracking; (2) Smooth surface formation with or without BP that depended on the stress concentration around inclusions and cracked Supergrain orientation in space according to acting external loading; (3) Drastically change to increasing of fracture surface roughness because of metals cracking by grain boundary of by slip bands; (4) Drastically change to fast fracture with dimpled surface formation.

From considered sequence of formed fracture surface areas one can be conclude that it is not only one way for metals deformation and destruction processes to realize fatigue crack propagation mechanisms on the all discussed stages. There can be intensive cracking by the grain before fast fracture without influence of this mechanism on the crack origination in one matrix. In another metal crack origination has been performed with supergrain but FSF took place in the area of origin.

Consequently it can be conclude that in the case of subsurface crack origination there takes place some specific selforganized process of matrix destruction that has to be investigated further more precisely to discover regularities in this process.

\section{QUANTUM-MECHANICAL NATURE OF PROPAGATED CRACKS}

I $\mathrm{n}$ the case of subsurface fatigue cracking it was introduced Paris-Hertzberg equation for metals in the next form [1]:

$$
d a / d N=b\left(\Delta K_{e f f} / E[b]^{1 / 2}\right)^{3}
$$

In Eq.(1), E is Young's modulus, b - modulus of Burger's vector.

Earlier it was theoretically considered crack growth process in metals based on non-continuous approach [6]. It was introduced knowledge about minimum value of crack increment such as quant of metals cracking, $a_{q}$, and this value has been calculated for steels [18] and Al-based alloys [19, 20]. Cascade of crack increments being more than $a_{q}-$ value was demonstrated and strongly expressed evidence of crack growth rate hierarchy was introduce in the next form:

$$
(d a / d N)_{i} /(d a / d N)_{i+1}=\left(\tau_{T} / \sigma_{T}\right)^{1 / q}
$$

In Eq. (2), $\tau_{T}$ and $\sigma_{T}$ are theoretical shear and tension stress, respectively, $q=1,2,4,8 \ldots$

Many experiments were done to confirm the relation (2) for Al-based alloys [19, 21], Fig.3. It was discovered constant values of crack growth rates in the crack growth direction with their hierarchy in accordance with Eq. (2).

Following by physical approach to metal failure it is very easy to shown that for all metals cannot be realized crack increment in one cycle less than two lattice. For, example, one dislocation has in homogeneity in two lattices at its tip, Fig.4. If sliding process is dominant ahead of a crack tip there will be interaction effect between two dislocations that directed to form inhomogeneities in the distance $4 \mathrm{~b}$ (see Fig.12b).

Values of $a_{q}$ were calculated and shown that for steels it is $0.5 \mathrm{~nm}$ [18], but for Al-based alloys it is 2nm [19], [21].

Quantum-mechanical nature of subsurface crack growth can be seen based on test results under tension of wide range of metals in area of infinitesimal deformations. It was discovered quantum-mechanical nature for quantization of Yang Modulus [22]. In the range of stress levels for metals subsurface cracking realizes so small deformations that they ought to be considered as metals extension in area of infinitesimal deformation. In this case it was shown that knowledge about elastic modulus disappeared because in this area of deformation exists quantization of modulus by the order [22]:

$$
E=2.06(1+v)(2 / 3)^{(s / 2)+(p / 4)} A\left[1-T /\left(2 T_{m}\right)\right]
$$




$$
\begin{aligned}
& A=2.89 \times 10^{5} \mathrm{MPa} \\
& s-1,2,3 \ldots, p=0(\text { or } 1) \\
& \mu(0)=(2 / 3)^{s / 2+p / 4} A
\end{aligned}
$$

In Eq.(3) $T_{m^{-}}$temperature of melting; $v$ - Poisson factor; - $\mu(0)$ shear modulus.

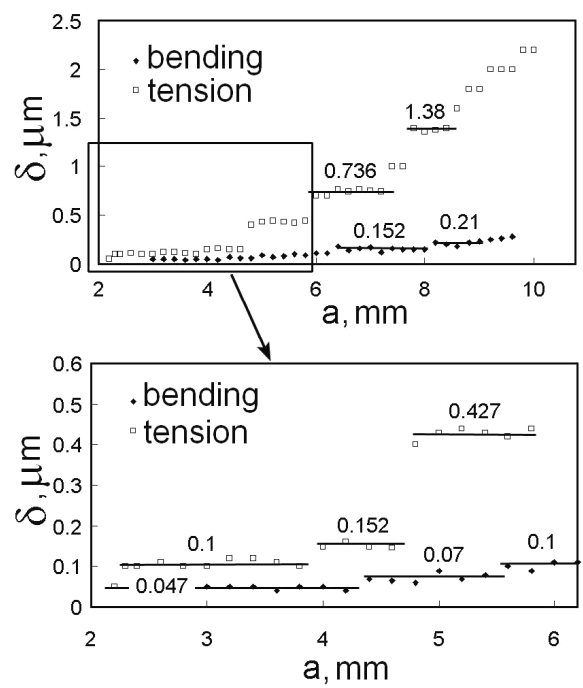

Figure 3: Fatigue striation spacing against crack growth in the depth direction for semi-elliptic crack in a specimen of AK6 Al-based alloy. Numbers indicate spacing-values calculated in accordance with Eq.2.

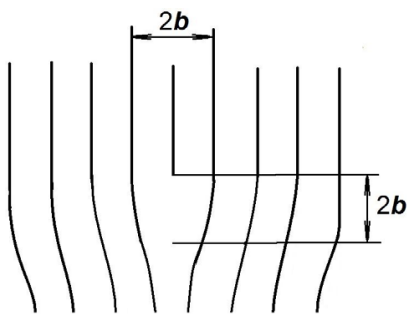

a)

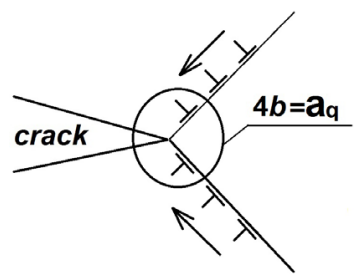

b)

Figure 4: Scheme of minimum inhomogeneity occurring (a) at the dislocation and (b) crack tip.

It is very clear that subsurface cracking in UHCF regime takes place with level of deformation being the same which have to be classified as infinitesimal deformations. That is why quantum-mechanical approach for consideration crack increments quantization has fundamental nature.

Another way of consideration for quantum-mechanical approach to fatigue crack growth can be introduced based on wellknown relation for time dependent processes of solid body degradation [23]:

$$
\tau=\tau_{0} \exp \left(U_{0}-\gamma \sigma\right) / k T
$$

In Eq.4 $\mathrm{U}_{0}$ - energy of activation for cracking process; $\gamma$ - constant; $\sigma$ - stress of external loading; $\tau$ - time for matrix cracking; $\tau_{0}$ - time for cracking start.

Let be considered crack growth rate in the form

$$
(d a / d N)_{i}=(d a / d \tau)=C \exp \left(-\left\{U_{0}-\gamma E \varepsilon\right\}_{i} / k T\right)
$$

In the case of infinitesimal deformations between stress and strain takes place not uniformly expressed dependence because of (6). Consequently, there will be cascade of crack growth rates constant values with constants energy levels $\left(U_{0}-\gamma E \varepsilon\right)_{i}$, where $\varepsilon_{i}$ is local deformation at the crack tip.

In the case of tests with high frequency measurements of crack increment can be realized now to register average value. That is why most of test results have not evidence of considered nature of crack growth. Nevertheless, in one of the well- 
known tests this effect has been demonstrated [2]. Horizontal line shows cascade of crack increments with constant value. Indeed, it cannot be exactly calculated values on constant increments but they evidently exist.

It needs to point out that during external stress level increasing there is in matrix process deformation with different value of elastic modulus for different areas in metals. That is why in matrix there will be different areas where plastic deformation will be realized. Difference in value of local yield stress under the same external stress level, for instance, for polycrystalline aluminum can be more than 1.5 times at the moment of its start. Under cyclic loading there will be difference in local stressing metals during uploading portion and unloading portion in each cycle because exist difference in elastic modulus for tension and compression too.

Moreover, the problem of specific environment low pressure for subsurface cracking shows that the $\left(\Delta K_{\text {eff }}\right)$ which has been used for crack growth rate calculation, ought to be diminished on the factor $\mathrm{Ce}=\left(\Delta K_{\mathrm{eff} f \mathrm{e}} /\left(\Delta K_{\mathrm{eff}}\right)_{\mathrm{se}}<1\right.$, where $(\Delta \mathrm{Keff})_{\mathrm{se}}$ is the stress intensity factor in environment with low pressure that at the one atmosphere. This consideration based on many test results that can be taken from review [21].

Summarizing performed briefly discussion of non-conventional metals subsurface fatigue cracking it ought to introduce corrections in Paris-Hertzberg [1] equation in the next form:

$$
d a / d N=a_{q}\left[C_{e} \Delta K_{e f f} / E\left(a_{q}\right)^{1 / 2}\right]^{3}
$$

For metals it should be considered physically quant of fracture $\mathrm{a}_{\mathrm{q}} \geq 2 \mathrm{~b}$. Parameter $C_{e}=\left(\Delta K_{e f f}\right)_{e} /\left(\Delta K_{e f f}\right)_{s e}<1$ has to be estimated for all metals applicably to results of fatigue cracking in vacuum and in environment.

Next point, that ought to be discussed, for process of subsurface matrix cracking is related to slope in Paris-Hertzberg equation (1). Earlier it was shown that for low range of crack increments in one cycle being less than $10^{-7} \mathrm{~m} / \mathrm{cycle}$, average value of crack growth rate can be considered under the load constant condition by the same manner that it is under constant deformation [21]. From this consideration follows that subsurface cracking takes place with slope in ParisHertzberg equation equals "2". This value of slope has been discovered in steels based on fatigue striations measurements for subsurface crack propagation [8]. The striation spacing interval was in the range of $(0.1-2.0) \times 10^{-6} \mathrm{~m}$. In this investigation has been discussed this situation and the discovered slope was equal "2" related to crack opening effect with cyclical plastic zone.

From the performed above consideration of subsurface crack propagation follows that it can be considered Paris equation in the next form:

$$
d a / d N=C_{o}\left(\Delta K_{e f f}\right)^{2}
$$

The value of $a_{q}$ has consideration at the threshold stress intensity factor, $\left(\Delta K_{e f f}\right)_{t b}$. That is why $C_{0}$ in Eq. (8) can be considered as $C_{0}=a_{q} /\left(\Delta K_{e f f}\right)_{t h}^{2}$. At the end we can conclude that

$$
d a / d N=a_{q}\left(\Delta K_{e f f} /\left[\Delta K_{e f f}\right]_{t b}\right)^{2}
$$

In the case of circular subsurface crack growing [10] the stress intensity factor formula is:

$$
\Delta K=(2 / \pi) \Delta \sigma[\pi a]^{1 / 2}
$$

Substituting (10) in (9) we can obtain simply equation:

$$
d a / d N=a_{q}\left(a_{c} / a_{0}\right)
$$

In Eq.6, $a_{0}$-value determines crack length at the FGA border with crack increment $a_{q}, a_{t}$-value determines maximum length of subsurface crack. This equation can be considered for failure analyses because there is not need in special knowledge of stress level or environment effect during subsurface cracking.

Calculated number of cycles by the formula (11) will be always less than real subsurface cracking period because FGA zone creation with spherical particles formation cannot be expressed in fracture mechanics terms.

\section{CONCLUDING REMARKS}

. New mechanism of fatigue crack subsurface origination was considered for metals with Fine-Granular-Area forming. First, this pattern has result of material hydrostatic pressing and rotation that directed to appearance ultrahigh-plasticity in a local volume with nano-structure performing. Second, fatigue cracking develops by the border of 
nano-structure with their rotation and spherical particles forming.

2. It was considered quantum-mechanical nature of metals fatigue cracking on nano-scale level and shown that crack increments in loading cycle have to be strongly expressed in accordance with introduced expression.

3. Simply equation was considered for subsurface cracking description and number of cycles' estimation. It can be used in

failure analyses of in-service cracked structures.

\section{REFERENCES}

[1] Bathias, C., Paris, C.P., Gigacycle fatigue in mechanical practice. Marcel Dekker, NY, USA, (2004).

[2] Sakai, T., Li, W., Lian, B., Oguma, N., Review and new analyses of fatigue crack initiation mechanisms of interior inclusion-induced fracture of high strength steels in very high cycle regime, in: C.Berger and H.-J.Christ (Eds.), Proc. of the Int. Conf. VHCF5, DVM, June 28-30, 2011, Berlin, Germany, (2011) 19-26.

[3] J.E., Jones, J.W., Larsen, J.M., Ritchie, R.O., Proc. of Fourth International Conference on Very High Cycle Fatigue, VHCF5, Edited by Allison, August 19-22, 2007. University of Michigan, Ann-Arbour, Michigan, USA, (2007).

[4] Shanyavskiy, A.A., Modelling of metals fatigue cracking. Synergetics in aviation. Ufa, Monograph, Russia, (2007).

[5] Karsch, T., Bomas, H., Zoch, H.-W., Influence of hydrogen and microstructure on the fatigue behavior of steel SAE 52100 in the VHCF regime, in: C.Berger and H.-J.Christ (Eds.), Proc. of the Int. Conf. VHCF5, Eds., DVM, June 2830, 2011, Berlin, Germany, (2011) 201-206.

[6] Nakamura, T., Oguma, H., Influence of environment on the formation unique morphology on fracture surface in subsurface fractures, in: C.Berger and H.-J.Christ (Eds.), Proc. of the Int. Conf. VHCF5, Eds., DVM, June 28-30, 2011, Berlin, Germany, (2011) 257-263

[7] Murakami, Yu., Metals Fatigue: Effects of Small Defects and Nonmetallic inclusions. Elsevier Ltd, London, UK, (2002).

[8] Shanyavskiy, A.A., Fatigue cracking of smooth and notched specimens of compacted superalloy EP741 NP in highand very-high-cycle-fatigue regime, in: C.Berger and H.-J.Christ (Eds.), Proc. of the Int. Conf. VHCF5, Eds., DVM, June 28-30, 2011, Berlin, Germany, (2011) 107-112.

[9] Carboni, M., Annoni, M., Ferraris, M., Analyses of premature failure of some aluminum alloy sonotrodes for ultrasonic welding, in: C. Berger and H.-J. Christ (Eds.), Proc. of the Int. Conf. VHCF5, Eds., DVM, June 28-30, 2011, Berlin, Germany, (2011) 589-594

[10] Huang, Z., Wagner, D., Bathias, C., Paris, C.P., Subsurface crack initiation and propagation mechanisms in gigacycle fatigue. Acta Materialia, 58 (2010) 6046-6054.

[11] Shanyavskiy, A., Banov, M., The twisting mechanism of subsurface fatigue cracking in Ti-6Al-2Sn-4Zr-2Mo-0.1Si alloy. Engineering Fracture Mechanics, 77 (2010) 1896-1906

[12] Vasiliyev, L.S., At the theory of metals plastic deformation with melted grain boundaries. Metals, 1 (2002) 112 - 122 (Russia)

[13] Wang, C., Nikitin, A., Shanyavskiy, A., Bathias, C., An understanding of crack growth in VHCF from an internal inclusion in high strength steel. in: Andrea Carpinteri, Andrea Spagnoli (Eds), Proceed. of the 4-th Intern. Conf. on “Crack Paths (CP 2012”), Gaeta (Italy), 19-21 September, (2012) 43.

[14] Shiozawa, K., Lu, L., Very high-cycle fatigue behaviour of shot-peened high-carbon chromium bearing steels. Fatigue Fract. Engng. Mater. Struct., 25 (2002) 813-822

[15] Karsch, T., Bomas, H., Zoch H.-W., Influence of hydrogen and microstructure on the fatigue behaviour of steel SAE 52100 in the VHCF regime. in: C.Berger and H.-J.Christ (Eds.), Proc. of the Int. Conf. VHCF5, Eds., DVM, June 2830, 2011, Berlin, Germany, (2011) 201-206.

[16] Shanyavskiy, A.A., Synergetical models of fatigue surface appearance in metals: the scale levels of self-organization the rotation effects, and density of fracture energy, in: G.N.Frantziskonis (Ed.) Proc. NATO "Advanced Research Workshop on PROBAMAT-21st Century", Tests, Models and Applications for the 21-st Century, Sept. 10-12 1997, Kluwer Academic Publisher (1998) 11-44.

[17] Shanyavskiy, A.A., Fatigue cracking simulation based on crack closure effects in Al-based sheet materials subjected to biaxial cyclic loads. Engineering Fracture Mechanics, Special issue, Multiaxial Fatigue, (Ed. by Andrea Carpinteri, Les P.Pook, Andrea Spagnoli), 78 (2011) 1516-1528

[18] Gurevich, S.E., Some aspects of fatigue fracture mechanics. In: Ivanova, V.S. and Gurevich, S.E. (Eds), Cyclical fracture toughness of metals and alloys, Nauka, Moscow, SSSR, (1981) 19-38. 
[19] Sasov, A.Yu., Shanyavsky, A.A., Fourier-fractography foundation of quantum mechanical nature of cracks growth. Acta Stereol., 6/III (1987) 825-830.

[20] Sasov A.Yu., Shanyavskiy, A.A., Processing of scanning electron microscope images on basis of fast Fouriertransformation. Scann. Microsc., 2 (1988) 827-834.

[21] Shanyavskiy, A.A., Tolerance fatigue cracking of aircraft structures. Synergetics in engineering applications. Ufa, Monograph, Russia, (2003).

[22] Bell, D.F., Mechanics of solids I, Encyclopedia Of Solid, Chief editor S.FLUGG, VIa/I, Springer Verlag, (1973).

[23] Jurkov, S.N., Problems of solids strength. Vest. Akad. Nauk USSR, 11 (1957) 78-82. 